\title{
Lifetime, mixing and CPV in ATLAS
}

\section{Malcolm Skinner ${ }^{* \dagger}$}

Lancaster University

E-mail: malcolm.bruce.skinnerecern.ch

The latest results measured by the ATLAS experiment on lifetime, mixing and $C P$ violation in the $B^{0}$ and $B_{s}^{0}$ systems are presented. First, the measurement of the $B_{s}^{0} \rightarrow J / \psi \phi$ decay parameters using $4.9 \mathrm{fb}^{-1}$ and $14.3^{-1}$ of integrated luminosity collected by the ATLAS detector at the LHC in $p p$ collisions at $\sqrt{s}=7 \mathrm{TeV}$ and $\sqrt{s}=8 \mathrm{TeV}$ respectively is given. The measured values include the $C P$-violating phase $\phi_{s}$ and the width difference of the mass eigenstates $\Delta \Gamma_{s}$. The measurements for the 7 and $8 \mathrm{TeV}$ data samples are combined, giving values of $\phi_{s}=-0.098 \pm 0.084$ (stat.) \pm 0.040 (syst.) rad and $\Delta \Gamma_{s}=0.083 \pm 0.011$ (stat.) \pm 0.007 (syst.) $\mathrm{ps}^{-1}$. The measured values agree with the Standard Model predictions. Second, the measurement of the relative width difference $\Delta \Gamma_{d} / \Gamma_{d}$ of the $B^{0}-\bar{B}^{0}$ system at $\sqrt{s}=7 \mathrm{TeV}$ and $\sqrt{s}=8 \mathrm{TeV}$, corresponding to an integrated luminosity of $25.2 \mathrm{fb}^{-1}$ is presented. The measured value is $\Delta \Gamma_{d} / \Gamma_{d}=(-0.1 \pm 1.1$ (stat.) \pm 0.9 (syst.) $) \times 10^{-2}$. Currently, this is the most precise single measurement of $\Delta \Gamma_{d} / \Gamma_{d}$. It agrees with the Standard Model prediction and measurements by other experiments.

16th International Conference on B-Physics at Frontier Machines

2-6 May 2016

Marseille, France

*Speaker.

$\dagger$ On behalf of the ATLAS collaboration. 


\section{The ATLAS detector}

ATLAS is a general purpose detector that conists of an inner tracker, a calorimeter and a muon spectrometer [1]. Analyses of $B$-physics in ATLAS mainly use information from the inner detector and the muon spectrometer. The inner detector provides momentum resolution in the pseudorapidity range $|\eta|<2.5$. The muon spectrometer provides muon identification and triggers. The ATLAS trigger system employs a Level-1 hardware trigger and two high-level software triggers. Heavy flavour analyses in ATLAS mostly use trigger selections based on a di-muon signature. These triggers have muon transverse momentum $\left(p_{T}\right)$ thresholds of 4 or $6 \mathrm{GeV}$ and pseudorapidity coverage of $|\eta|<2.4$. Di-muon vertex reconstruction is also utilized.

\section{2. $C P$ violation in the $B_{s}^{0} \rightarrow J / \psi \phi$ decay}

The occurence of $C P$ violation in the $B_{s}^{0}$ system is due to interference between direct decays and decays with $B_{s}^{0}-\bar{B}_{s}^{0}$ mixing to a final state accessible to both $B_{s}^{0}$ and $\bar{B}_{s}^{0}$, such as $J / \psi \phi$. The $C P$ asymmetry is represented by the weak phase difference $\phi_{s}$ between the $B_{s}^{0}-\bar{B}_{s}^{0}$ mixing amplitude and the $b \rightarrow c \bar{c} s$ decay amplitude. In the Standard Model, $\phi_{s}$ is predicted to be small and is related to elements of the CKM matrix [2]:

$$
\phi_{s} \approx-2 \beta_{s}=2 \arg \left(-\frac{V_{t s} V_{t b}^{*}}{V_{c s} V_{c b}^{*}}\right)=-0.0363_{-0.0015}^{+0.0016} \mathrm{rad} .
$$

The keen interest in $B_{s}^{0} \rightarrow J / \psi \phi$ is because of the possible new physics contribution that may exist within $\phi_{s}$, which can therefore be expressed as the sum of its SM and NP components:

$$
\phi_{s}=\phi_{s}^{S M}+\phi_{s}^{N P}
$$

Precise measurements of $\phi_{s}$ performed by LHC experiments have already constrained this new physics contribution, but more precision is still needed to test the SM and evaluate $\phi_{s}^{N P}$.

The results of the $B_{s}^{0} \rightarrow J / \psi \phi$ analysis using $4.9 \mathrm{fb}^{-1}$ of integrated luminosity collected by ATLAS in 2011 at $\sqrt{s}=7 \mathrm{TeV}$ were published in 2014 [3]. The measured values of $\phi_{s}$ and the width difference $\Delta \Gamma_{s}$ were:

$$
\begin{array}{r}
\phi_{s}=0.12 \pm 0.25 \text { (stat.) } \pm 0.05 \text { (syst.) rad. } \\
\Delta \Gamma_{s}=0.053 \pm 0.21 \text { (stat.) } \pm 0.010 \text { (syst.) } \mathrm{ps}^{-1} .
\end{array}
$$

The more recent analysis of the $14.3 \mathrm{fb}^{-1}$ of integrated luminosity collected in 2012 at $\sqrt{s}=8$ $\mathrm{TeV}$ was released by the ATLAS collaboration in January 2016 [4]. In addition to the increased data sample, this analysis included a number of improvements over the 2011 analysis.

The $B_{s}^{0} \rightarrow J / \psi \phi$ candidates were selected by fitting the four track combinations (two muon tracks and two hadronic tracks) to a common vertex. The muon tracks were required to pass the trigger thresholds of $p_{T}>4$ or $6 \mathrm{GeV}$ and $\eta<|2.4|$. The hadronic tracks were required to be oppositely charged and have $p_{T}>1 \mathrm{GeV}$ and $\eta<|2.5|$. All selectrion criteria are independent of the $B_{s}^{0}$ lifetime. A total of $375987 B_{s}^{0}$ candidates were selected with $5.15<m(J / \psi \phi)<5.65$. The 
number of signal candidates was estimated to be $74900 \pm 400$, which is approximately 3.5 times that of the sample used in the 2011 analysis.

Since the measured effect in the $B_{s}^{0} \rightarrow J / \psi \phi$ analysis is due to $B_{s}^{0}-\bar{B}_{s}^{0}$ mixing, it is important to tag the initial flavour of the $B_{s}^{0}$ or $\bar{B}_{s}^{0}$ meson. Opposite side flavour tagging is used in ATLAS. The flavour tagging algorithm is calibrated using $B^{+} \rightarrow J / \psi K^{ \pm}$events. Three flavour tagging methods are used: opposite side muon tagging, opposite side electron tagging and jet charge tagging. The jet charge tag uses the jet with largest value of $b$-tagging in the event, excluding the $B_{s}^{0}$ decay. The tagging variable is given by:

$$
Q=\frac{\sum_{i} q^{i}\left(p_{T}^{i}\right)^{k}}{\sum_{i}\left(p_{T}^{i}\right)^{k}}
$$

where $q^{i}$ and $p_{T}^{i}$ are, respectively, the charge and transverse momentum of track $i$. For muon (electron) tagging, $k=1.1(1.0)$ and the sum is over tracks within a cone of $\Delta R<0.5$ around the muon (electron). For jet charge tagging, $k=1.1$ and the sum is over all tracks in the jet.

An unbinned maximum likelihood fit is used to extract the values of the physical parameters of the $B_{s}^{0} \rightarrow J / \psi \phi$ decay. The information used by the fit includes several variables describing the $B_{s}^{0} \rightarrow J / \psi \phi$ candidates including the reconstructed mass, transverse momentum, proper decay time, the measured proper decay time uncertainty, transversity angles and flavour tagging value. The maximum likelihood fit of the $8 \mathrm{TeV}$ data sample gives the following results for $\phi_{s}$ and $\Delta \Gamma_{s}$ :

$$
\begin{aligned}
\phi_{s} & =-0.123 \pm 0.089 \text { (stat.) } \pm 0.041 \text { (syst.) rad. } \\
\Delta \Gamma_{s} & =0.096 \pm 0.013 \text { (stat.) } \pm 0.007 \text { (syst.) } \mathrm{ps}^{-1} \text {. }
\end{aligned}
$$

The results from the $7 \mathrm{TeV}$ and $8 \mathrm{TeV}$ data samples are consistent and are combined:

$$
\begin{aligned}
\phi_{s} & =-0.098 \pm 0.084 \text { (stat.) } \pm 0.040 \text { (syst.) rad. } \\
\Delta \Gamma_{s} & =0.083 \pm 0.011 \text { (stat.) } \pm 0.007 \text { (syst.) } \mathrm{ps}^{-1} .
\end{aligned}
$$

Figure 1 shows the likelihood contours in the $\phi_{s}-\Delta \Gamma_{s}$ plane for the $7 \mathrm{TeV}$ and $8 \mathrm{TeV}$ analyses seperately and combined.

\section{Measurement of the relative width difference of the $B^{0}-\bar{B}^{0}$ system}

The relative value of the $B^{0}-\bar{B}^{0}$ width difference $\Delta \Gamma_{d} / \Gamma_{d}$ is reliably predicted in the Standard Model [5]:

$$
\Delta \Gamma_{d} / \Gamma_{d}=(0.42 \pm 0.08) \times 10^{-2}
$$

It has been shown [6] that a relatively large variation of $\Delta \Gamma_{d} / \Gamma_{d}$ due to a possible new physics contribution would not contradict other existing SM results. A precise measurement of $\Delta \Gamma_{d} / \Gamma_{d}$ would therefore provide a stringent test of the underlying theory, complementery to other searches.

The current experimental uncertainty of $\Delta \Gamma_{d} / \Gamma_{d}$ is much larger than the SM central value, which prevents a meaningful test of the SM prediction. Furthermore, the measurements of $\Delta \Gamma_{d} / \Gamma_{d}$ made by Belle [7] and LHCb [8] differ by more than 1.5 standard deviations. Therefore, more precise measurements of $\Delta \Gamma_{d} / \Gamma_{d}$ are needed to establish its value and perform an important test of the SM. The measurement of $\Delta \Gamma_{d} / \Gamma_{d}$ was performed by the ATLAS collaboration using he methods described below. The full details of this analysis are given in the corresponding paper [9]. 


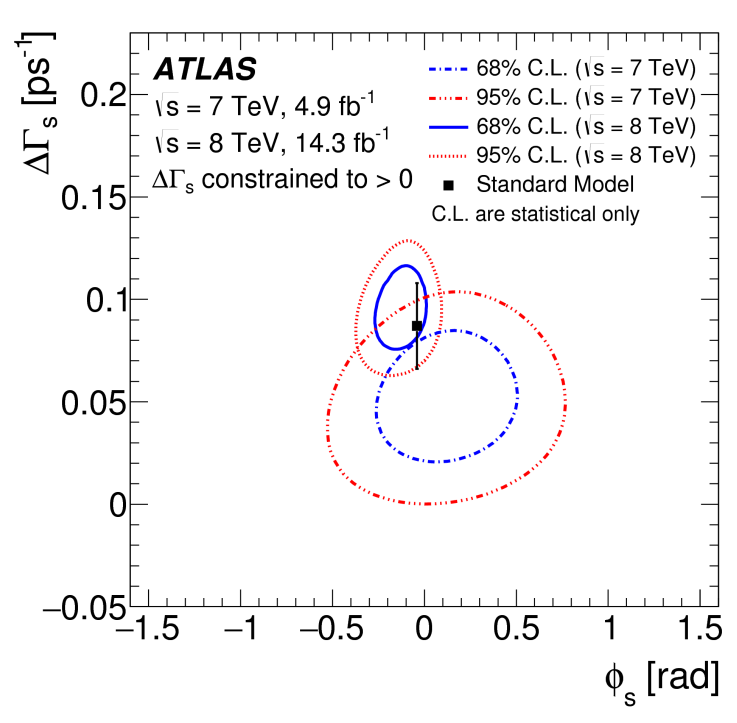

(a)

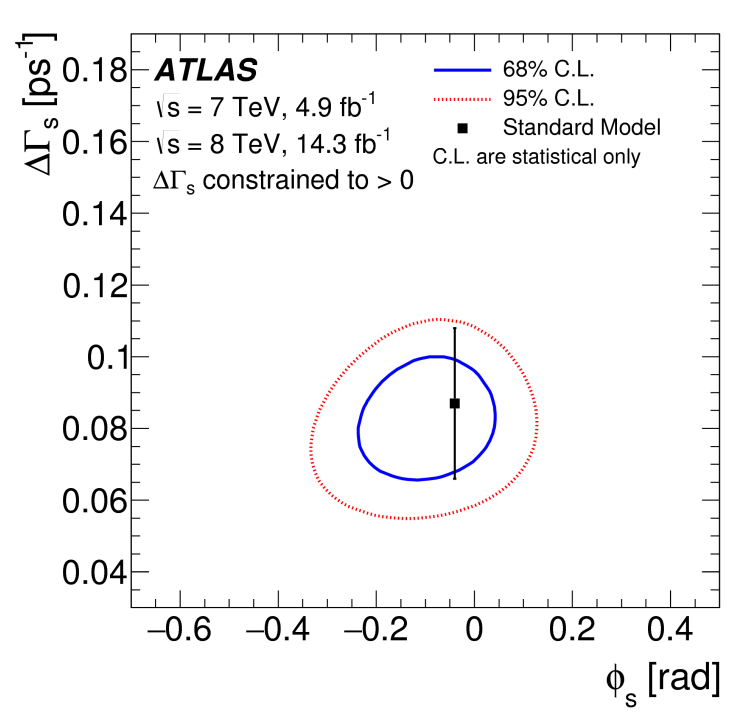

(b)

Figure 1: Likelihood contours in the $\phi_{s}-\Delta \Gamma_{s}$ plane for (a) the seperate results and (b) the combined results from the $\sqrt{s}=7 \mathrm{TeV}$ and $\sqrt{s}=8 \mathrm{TeV}$ analyses [4]. The blue line shows the $68 \%$ likelihood contour, while the red dotted line shows the $95 \%$ likelihood contour (statistical errors only).

The untagged time-dependent decay rate of $B^{0}$ meson into a final state $f$ is given by:

$$
\Gamma(f, t) \propto \mathrm{e}^{-\Gamma_{d} t}\left[\cosh \frac{\Delta \Gamma_{d} t}{2}+A_{p} A_{\mathrm{CP}}^{\mathrm{dir}} \cos \left(\Delta m_{d} t\right)+A_{\Delta \Gamma} \sinh \frac{\Delta \Gamma_{d} t}{2}+A_{p} A_{\mathrm{CP}}^{\operatorname{mix}} \sin \left(\Delta m_{d} t\right)\right] .
$$

In this expression, $\Gamma_{d}$ and $\Delta m_{d}$ are respectively the mean width and mass difference of the $B^{0}-\bar{B}^{0}$ system. The parameters $A_{\mathrm{CP}}^{\mathrm{dir}}, A_{\Delta \Gamma}$ and $A_{\mathrm{CP}}^{\mathrm{mix}}$ depend on the final state $f$. The abbreviations "dir" and "mix" stand for "direct" and "mixing". By definition, $\left|A_{\mathrm{CP}}^{\mathrm{dir}}\right|^{2}+\left|A_{\Delta \Gamma}\right|^{2}+\left|A_{\mathrm{CP}}^{\mathrm{mix}}\right|^{2} \equiv 1$. The final states considered in this analysis were $J / \psi K_{S}$ and $J / \psi K^{* 0}$. The $J / \psi$ is reconstructed using the decay $J / \psi \rightarrow \mu^{+} \mu^{-}$. The $K_{S}$ and $K^{* 0}$ are reconstructed using the $K_{S} \rightarrow \pi^{+} \pi^{-}$and $K^{* 0} \rightarrow K^{+} \pi^{-}$ decay modes. For the $J / \psi K^{* 0}\left(\bar{K}^{* 0}\right)$ final state, $A_{\mathrm{CP}}^{\mathrm{dir}}=+1(-1), A_{\Delta \Gamma}=0, A_{\mathrm{CP}}^{\mathrm{mix}}=0$. For the $J / \psi K_{S}$ channel, $A_{\mathrm{CP}}^{\mathrm{dir}}=0, A_{\Delta \Gamma}=\cos 2 \beta, A_{\mathrm{CP}}^{\mathrm{mix}}=-\sin 2 \beta$, where $\beta$ is the Unitarity Triangle angle measured as $\sin 2 \beta=0.679 \pm 0.020$ [10]. $A_{P}$ is the production asymmetry of $B^{0}$ and $\bar{B}^{0}$ in ATLAS.

The value of $\Delta \Gamma_{d} / \Gamma_{d}$ is determined by measuring the experimental ratio of the proper decay length $\left(L_{\text {prop }}^{B}\right)$ distributions of the two decay modes. The sensitivity to $\Delta \Gamma_{d} / \Gamma_{d}$ comes from $\Gamma\left(J / \psi K_{S}, L_{\text {prop }}^{B}\right)$ while $\Gamma\left(J / \psi K^{* 0}, L_{\text {prop }}^{B}\right)$ provides the normalization, which cancels the factor of $\mathrm{e}^{-\Gamma_{d} t}$, increasing the sensitivity to $\Delta \Gamma_{d} / \Gamma_{d}$. This method also helps to reduce come of the systematic uncertainties.

The proper decay length distribution is obtained by first dividing the range of $L_{\text {prop }}^{B}$ between -0.3 and $6.0 \mathrm{~mm}$ into ten bins. In each bin, distributions of the invariant mass of the $J / \psi K_{S}$ and $J / \psi K^{* 0}$ candidates are produced and the number of signal $B^{0} \rightarrow J / \psi K_{S}$ and $B^{0} \rightarrow J / \psi K^{* 0}$ candidates in each bin is determined by a fit to these distributions. The total number of $B^{0} \rightarrow J / \psi K_{S}$ decays is $28170 \pm 250$ in the $7 \mathrm{TeV}$ data sample and $110830 \pm 520$ in the $8 \mathrm{TeV}$ data sample. 
The total number of $B^{0} \rightarrow J / \psi K^{* 0}$ candidates is $129200 \pm 900$ in the $7 \mathrm{TeV}$ data sample and $555800 \pm 1900$ in the $8 \mathrm{TeV}$ sample.

The ratio of the number of $B^{0}$ candidates in the two channels in each $L_{\text {prop }}^{B}$ bin gives the experimental ratio of proper decay lengths $R_{i, \text { uncor }}\left(L_{\text {prop }}^{B}\right)$. This ratio must be corrected to account for the difference in reconstruction efficiencies of the $B^{0} \rightarrow J / \psi K_{S}$ and $B^{0} \rightarrow J / \psi K^{* 0}$ channels. This difference exists because the hadronic tracks in the $B^{0} \rightarrow J / \psi K_{S}$ decay come from a displaced $K_{S} \rightarrow \pi \pi$ vertex, while all four tracks from the $B^{0} \rightarrow J / \psi K^{* 0}$ decay come from a single vertex. This difference is the largest source of experimental bias in $R_{i, \text { uncor }}\left(L_{\text {prop }}^{B}\right)$ and it can be assessed only with Monte Carlo simulation. The ratio of reconstruction efficiencies in MC in each $L_{\text {prop }}^{B}$ bin is determined:

$$
R_{i, \text { eff }}\left(L_{\text {prop }}^{B}\right)=\frac{\varepsilon_{i}\left(J / \psi K_{S}, L_{\text {prop }}^{B}\right)}{\varepsilon_{i}\left(J / \psi K^{* 0}, L_{\text {prop }}^{B}\right)} .
$$

The ratio $R_{i \text {,uncor }}\left(L_{\text {prop }}^{B}\right)$ is divided by $R_{i, \text { eff }}\left(L_{\text {prop }}^{B}\right)$ to obtain the corrected ratio $R_{i, \text { cor }}\left(L_{\text {prop }}^{B}\right)$.

The $B^{0}-\bar{B}^{0}$ production asymmetry is measured from the charge asymmetry of the $B^{0} \rightarrow$ $J / \psi K^{* 0}$ decay, which is measured as a function of $L_{\text {prop }}^{B}$. The charge asymmetry has two contributions: The detector asymmetry $A_{\mathrm{det}}$ and the production asymmetry $A_{P}$ which should oscillate with $L_{\text {prop }}^{B}$. The values of $A_{\text {det }}$ and $A_{P}$ measured by the ATLAS experiment using data obtained at 7 and $8 \mathrm{TeV}$ are:

$$
\begin{aligned}
A_{\text {det }} & =(+1.33 \pm 0.24 \pm 0.30) \times 10^{-2} . \\
A_{P} & =(+0.25 \pm 0.48 \pm 0.05) \times 10^{-2} .
\end{aligned}
$$

The first uncertainty is statistical and the second is due to uncertainties in the mistag fraction of $K^{* 0}$ and $\bar{K}^{* 0}$ and in the value of $|q / p|$.

The corrected ratio of proper decay lengths $R_{i, \text { cor }}\left(L_{\text {prop }}^{B}\right)$ is fitted by the expected number of events in each channel, in each bin. The fitted distributions of $R_{i, \text { cor }}\left(L_{\text {prop }}^{B}\right)$ for the 7 and $8 \mathrm{TeV}$ data samples are shown in Fig. 2. The values of $\Delta \Gamma_{d} / \Gamma_{d}$ at $\sqrt{s}=7 \mathrm{TeV}$ and $\sqrt{s}=8 \mathrm{TeV}$ are obtained seperately:

$$
\begin{array}{ll}
\left.\Delta \Gamma_{d} / \Gamma_{d}=(-2.8 \pm 2.2 \text { (stat.) } \pm 1.7 \text { (syst. })\right) \times 10^{-2} & {[\sqrt{s}=7 \mathrm{TeV}]} \\
\left.\Delta \Gamma_{d} / \Gamma_{d}=(+0.8 \pm 1.3 \text { (stat.) } \pm 0.8 \text { (syst. })\right) \times 10^{-2} & {[\sqrt{s}=8 \mathrm{TeV}]}
\end{array}
$$

The results from the two data samples are consistent and are combined:

$$
\left.\left.\Delta \Gamma_{d} / \Gamma_{d}=(-0.1 \pm 1.1 \text { (stat. }) \pm 0.9 \text { (syst. }\right)\right) \times 10^{-2}
$$

\section{Conclusions}

The measurement of $C P$ violation in the $B_{s}^{0} \rightarrow J / \psi \phi$ using data collected by the ATLAS experiment during Run 1 of the LHC is consistent with measurements by other experiments. It also agrees with the SM prediction. There is, however, still room for new physics in $C P$ violation in this channel.

The value of $\Delta \Gamma_{d} / \Gamma_{d}$ obtained by ATLAS during LHC Run 1 is currently the most precise measurement of this quantity. The result agress with the SM prediction. It is also consistent measurements performed by other experiments. 


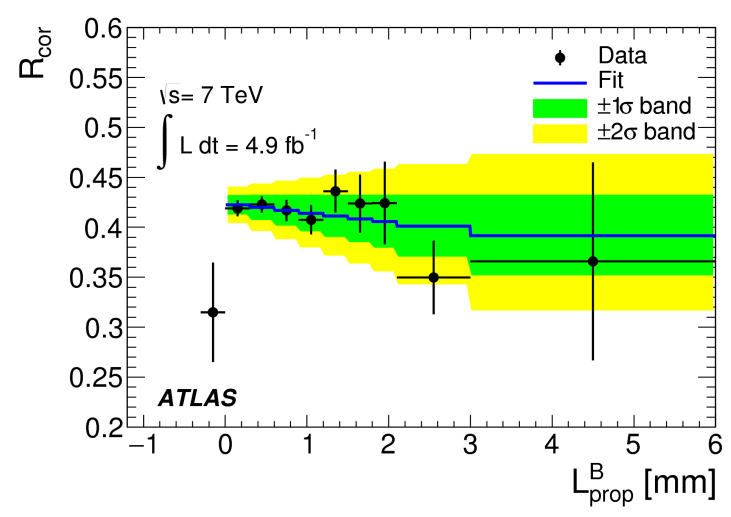

(a)

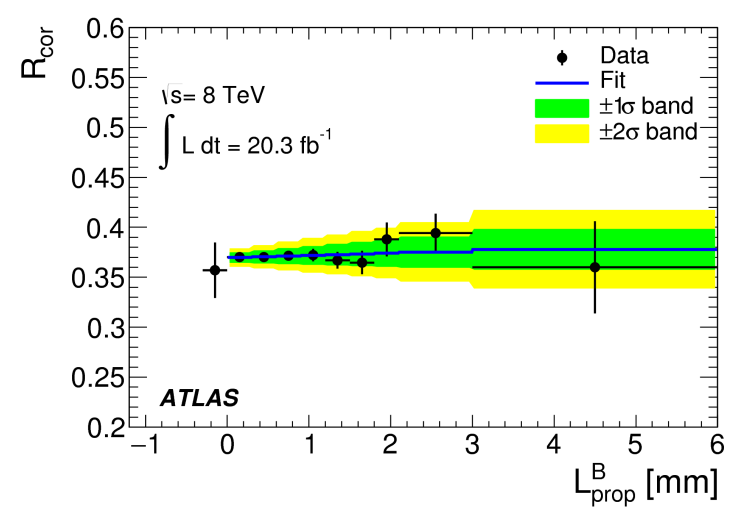

(b)

Figure 2: Efficiency-corrected ratio of the observed decay length distributions, $R_{\text {cor }}\left(L_{\text {prop }}^{B}\right)$ for the (a) $\sqrt{s}=7$ $\mathrm{TeV}$ and (b) $\sqrt{s}=8 \mathrm{TeV}$ data samples [9]. The normalisation of the two data sets is arbitrary. The full line shows the fit of $R_{\text {cor }}\left(L_{\text {prop }}^{B}\right)$. The error bands correspond to uncertainties in $\Delta \Gamma_{d} / \Gamma_{d}$ determined by the fit.

\section{References}

[1] ATLAS Collaboration, The ATLAS Experiment at the CERN Large Hadron Collider, JINST 3 (2008) S08003.

[2] J. Charles et al., Predictions of selected flavor observables within the standard model, Phys. Rev. D 84 (2011) 033005.

[3] ATLAS Collaboration, Flavor tagged time-dependent angular analysis of the $B_{s} \rightarrow J / \psi \phi$ decay and extraction of $\Delta \Gamma$ s and the weak phase $\phi_{s}$ in ATLAS, Phys. Rev. D 90 (2014) no.5, 052007, arXiv:1407.1796 [hep-ex].

[4] ATLAS Collaboration, Measurement of the CP-violating phase $\phi_{s}$ and the $B_{s}^{0}$ meson decay width difference with $B_{s}^{0} \rightarrow J / \psi \phi$ decays in ATLAS, arXiv:1601.03297 [hep-ex].

[5] A. Lenz and U. Nierste, Numerical Updates of Lifetimes and Mixing Parameters of B Mesons, arXiv:1102.4274 [hep-ph].

[6] C. Bobeth, U. Haisch, A. Lenz, B. Pecjak and G. Tetlalmatzi-Xolocotzi, On new physics in $\Delta \Gamma_{d}$, JHEP 1406 (2014) 040 arXiv:1404.2531 [hep-ph].

[7] T. Higuchi et al. [Belle Collaboration], Search for Time-Dependent CPT Violation in Hadronic and Semileptonic B Decays, Phys. Rev. D 85 (2012) 071105 arXiv:1203.0930 [hep-ex]

[8] R. Aaij et al. [LHCb Collaboration], Measurements of the $B^{+}, B^{0}, B_{s}^{0}$ meson and $\Lambda_{b}^{0}$ baryon lifetimes, JHEP 1404 (2014) 114 arXiv:1402.2554 [hep-ex]

[9] ATLAS Collaboration, Measurement of the relative width difference of the $B^{0}-\bar{B}^{0}$ system with the ATLAS detector, arXiv:1605.07485 [hep-ex].

[10] K.A. Olive and Particle Data Group, Review of Particle Physics Chinese Physics C 38(9) (2014) 090001. 